\title{
Moving Toward a More Informed Approach to Risk Stratification of Patients: Comments on Seror et al. CT-Derived Liver Surface Nodularity and Sarcopenia as Prognostic Factors in Patients with Resectable Metabolic Syndrome-Related HCC
}

\author{
Susan Tsai, MD ${ }^{1}$, and Timothy M. Pawlik, MD, MPH, MTS, $\mathbf{P h D}^{2}$ (D) \\ ${ }^{1}$ Department of Surgery, Medical College of Wisconsin, Milwaukee, WI; ${ }^{2}$ Department of Surgery, The Urban Meyer III \\ and Shelley Meyer Chair for Cancer Research, The Ohio State University Wexner Medical Center, Columbus, OH
}

Currently, up to $20 \%$ of hepatocellular carcinoma (HCC) cases in the Western World may be attributed to nonalcoholic fatty liver disease (NAFLD) and metabolic syndrome. ${ }^{1}$ The relative incidence of NAFLD-associated $\mathrm{HCC}$ is anticipated to rise as the global obesity and diabetes epidemic worsen; in contrast, viral-associated HCC has decreased with access to effective antiviral treatments. The surgical management of patients with metabolic syndrome-related HCC can be challenging given the increased risk associated with obesity, cardiovascular disease, and diabetes with overall morbidity, as well as underlying liver dysfunction. ${ }^{2}$ Although existing nomograms have been utilized to risk stratify surgical patients with varying degrees of success, procedure- and disease-specific tools have not been as developed. ${ }^{3}$ Novel tools, therefore, are needed to refine risk estimation among patients being considered for hepatic resection for different indications.

Mirroring the rise of precision medicine in which molecular determinants of cancers have been used to define prognosis and identify new therapeutics, there is growing interest in the field of radiomics. Radiomics capitalizes on the enormous amount of data available from medical imaging to transform qualitative visual assessments into reproducible quantitative measurements. ${ }^{4}$ Taken one step

(C) Society of Surgical Oncology 2020

First Received: 28 August 2020

Accepted: 3 September 2020;

Published Online: 20 September 2020

T. M. Pawlik, MD, MPH, MTS, PhD

e-mail: tim.pawlik@osumc.edu further, radiogenomics utilizes quantitative subvisual imaging features to build phenotypic-genotypic models down to a molecular level. The early adoption of radiomics was pioneered in lung cancer and has been used to predict distant metastases, as well as risk stratify patients for adjuvant therapy. ${ }^{5,6}$ Of note, radiomic data have been effective at characterizing and quantifying not only diseased tissues, but this technique also can help to quantitate and assess nondiseased, "normal" tissues. To this point, assessment of tumor versus nontumor tissue is extremely important among patients with HCC, wherein the surgeon must evaluate the tumor relative to the underlying quality and quantity of the nontumorous hepatic parenchyma to make appropriate clinical decisions.

The current study by Seror et al. examined two discrete radiologic features in a population of patients with metabolic syndrome/NAFLD who underwent liver resection for HCC. Liver surface nodularity (LSN) and absence of lean body mass (skeletal muscle index) were chosen as surrogate measures of liver cirrhosis and physiologic frailty, respectively. LSN is a measurement of surface liver contour irregularities, which has previously been demonstrated to be accurate at discriminating stages of hepatic fibrosis. ${ }^{7}$ Skeletal muscle index is an estimation of sarcopenia that is a surrogate for physiologic frailty. Several previous reports have demonstrated that a lower skeletal muscle index was associated with higher risk of postoperative complications among patients with primary liver tumors undergoing hepatectomy or liver transplantation. ${ }^{8,9}$ Given the known association between metabolic syndrome and sarcopenia, it was somewhat surprising that only $24 \%$ of the cohort had sarcopenia-a prevalence that was no different than the 
prevalence observed in a more general population of patients with primary liver tumors. ${ }^{9}$ The study did confirm the known association of LSN with histologic evidence of fibrosis. ${ }^{7}$ More interestingly, the study examined the impact of sarcopenia and LSN on postoperative outcomes. Specifically, among the 110 patients with metabolic syndrome, $34(30 \%)$ patients experienced a severe complication following hepatectomy. In the adjusted model, higher LSN scores [odds ratio (OR) 7.05, 95\% confidence interval (CI) 2.13-23.35] and sarcopenia (OR 6.51, 95\% CI 2.08-20.39) were associated with a marked increase in the risk of severe complications. Unfortunately, the small sample size resulted in very large point estimates and extremely wide $95 \%$ confidence intervals, suggesting imprecise estimates relative to the "true" effect of LSN and sarcopenia on postoperative complications. As such, the limited numbers of patients who had the "event" of interest (e.g., severe complication) was a major limitation of the current study. In turn, when the authors attempted to examine the multidimensional score that included both hepatic and global physiologic vulnerabilities, the analyses similarly suffered from low numbers. Notwithstanding this limitation, it was interesting to note that the composite assessment of both LSN and sarcopenia seemed to be associated with an even higher risk of complications versus patients with no LNS or sarcopenia (71\% vs. $7 \%$, $p<0.001)$. While somewhat preliminary in nature, data from the study did provide an early signal to highlight the potential value of incorporating radiomic machine-learning algorithms into clinical decision-making. Specifically, models that incorporate patient-sprcific (i.e., sarcopenia) with disease-specific (i.e., LSN) information may more accurately reflect the complex relationship between host physiology and tumor biology.

Unfortunately, while a wealth of data has been published on the association of low skeletal muscle index with adverse postoperative outcomes, little progress has been made to mitigate this risk ${ }^{9-13}$ In part, the lack of progress may reflect a more fundamental challenge in understanding the etiology of skeletal muscle atrophy, as well as an inability to classify "muscle wasting" as cancer cachexia versus noncancer-related sarcopenia. While Seror et al. did not differentiate "true" sarcopenia from cachexia, the clinical definitions of each are distinct. In particular, the distinction is important as sarcopenia may be reversible, while cancer cachexia is almost always due to an irreversible physiologic decline. ${ }^{14}$ In contrast to cachexia, which involves loss of both muscle mass and fat tissue, sarcopenia is more multidimensional and denotes both loss of muscle mass and muscle function (as measured by hand grip strength, walking speed, etc.). ${ }^{14,15}$ To date, most studies examining low skeletal mass index relative to postoperative complications have been retrospective and have failed to include any functional assessment. The "best" cutoff value to define sarcopenia using any index also has not been well-defined. Of note, Seror et al. did not report patient functional data, nor was the cut-off for the skeletal muscle index defined rigorously. Current consensus guidelines from the European Working Group on Sarcopenia in Older People recommended cutoff values based on normative data within any given study population. In fact, the prevalence of sarcopenia varies widely by imaging characteristics depending on the population and the cutoffs used. ${ }^{15}$ The original skeletal muscle index cutpoint proposed by Prado et al. was based on $\mathrm{CT}$ imaging ( $<38.5 \mathrm{~cm} / \mathrm{m}^{2}$ for females and $<52.4 \mathrm{~cm} / \mathrm{m}^{2}$ for males), while the most recent recommendations from the National Institutes of Health define skeletal muscle index based on dual-energy x-ray absorptiometry (DXA). ${ }^{8}$ The lack of cutoff values underlines the potential for misclassification, as well as the need to standardize reference populations. ${ }^{16}$

In the study by Seror et al., LSN was acquired using a semiautomated CT software tool that created a reference line to mimic the expected normal liver surface; variance from the expected line was detected from continuous CT images using a process that was replicated multiple times. In contrast to measuring skeletal muscle index, which can be time and labor intensive, the LSN methodology required minimal time or expertise. As a result, LSN has been applied to large cohorts in an attempt to examine its association with pathologic endpoints (hepatic fibrosis) and clinical endpoints (hepatic decompensation). ${ }^{7,17}$ LSN has demonstrated discrimination over a range of fibrosis scores and the discrimination improved with increasing fibrosis scores, suggesting that radiomic data can accurately predict meaningful biologic differences. In the study by Seror et al., high LSN scores were associated with recurrencefree survival, but not overall survival. The association with recurrence-free, yet not overall survival, was puzzling and hard to interpret. While the incongruent findings may have been related to differences in treatment after recurrence, data regarding treatment for recurrent disease was not reported. Nevertheless, the authors postulated that the association of LSN with fibrosis reflected underlying biology of the remnant liver that may have correlated with increased risk of recurrence. The relationship between tissue phenotype (liver nodularity) and underlying cellular and molecular changes is a fundamental premise of radiogenomics. A recent radiogenomic study in patients with HCC demonstrated an association of radiomic features with immune infilatration. ${ }^{18}$ Qualitative and quantitative textural analyses based on MRI images correlated with infiltrating immune cells, as well as mRNA expression of PD-L1 and CTLA4. This work suggested that it may be feasible to identify molecular radiomic signatures to serve as important relevant actionable targets. Similar to 
next generation sequencing data, these types of analyses require extensive bioinformatic and biostatistical expertise. In particular, automation with deep learning algorithms and artificial intelligence may be necessary to identify optimal radiomic patterns.

In summary, the study by Seror et al. had several limitations, yet provided cause for cautious optimism related to the utility of radiomics in assessing host and tumor risk factors related to patients undergoing surgery for HCC. Future challenges will involve standardization of methodologic protocols to eliminate misclassification, as well as expansion of radiomic features beyond simple binary associations of radiographic and clinical data points. Rather, combining advanced noninvasive imaging technology with state-of-the-art data analytics is needed to develop comprehensive functional and biologic algorithms to better implement and deliver precision medicine.

DISCLOSURES The authors declare no conflicts of interest.

\section{REFERENCES}

1. Younossi ZM, Koenig AB, Abdelatif D, Fazel Y, Henry L, Wymer M. Global epidemiology of nonalcoholic fatty liver disease: meta-analytic assessment of prevalence, incidence, and outcomes. Hepatology. 2016;64(1):73-84.

2. Bhayani NH, Hyder O, Frederick W, et al. Effect of metabolic syndrome on perioperative outcomes after liver surgery: a National Surgical Quality Improvement Program (NSQIP) analysis. Surgery. 2012;152(2):218-26.

3. Bilimoria KY, Liu Y, Paruch JL, et al. Development and evaluation of the universal ACS NSQIP surgical risk calculator: a decision aid and informed consent tool for patients and surgeons. $J$ Am Coll Surg. 2013;217(5):833-42 e831-3.

4. Lambin P, Leijenaar RTH, Deist TM, et al. Radiomics: the bridge between medical imaging and personalized medicine. Nat Rev Clin Oncol. 2017;14(12):749-62.

5. Coroller TP, Grossmann P, Hou Y, et al. CT-based radiomic signature predicts distant metastasis in lung adenocarcinoma. Radiother Oncol. 2015;114(3):345-50.

6. Vaidya P, Bera K, Gupta A, et al. CT derived radiomic score for predicting the added benefit of adjuvant chemotherapy following surgery in Stage I, II resectable Non-Small Cell Lung Cancer: a retrospective multi-cohort study for outcome prediction. Lancet Digit Health. 2020;2(3):e116-28.

7. Pickhardt PJ, Malecki K, Kloke J, Lubner MG. Accuracy of liver surface nodularity quantification on MDCT as a noninvasive biomarker for staging hepatic fibrosis. AJR Am J Roentgenol. 2016;207(6):1194-9.

8. Prado CM, Lieffers JR, McCargar LJ, et al. Prevalence and clinical implications of sarcopenic obesity in patients with solid tumours of the respiratory and gastrointestinal tracts: a population-based study. Lancet Oncol. 2008;9(7):629-35.

9. Valero V, 3rd, Amini N, Spolverato G, et al. Sarcopenia adversely impacts postoperative complications following resection or transplantation in patients with primary liver tumors. $J$ Gastrointest Surg. 2015;19(2):272-81.

10. Peng PD, van Vledder MG, Tsai S, et al. Sarcopenia negatively impacts short-term outcomes in patients undergoing hepatic resection for colorectal liver metastasis. $H P B$. 2011;13(7):439-46.

11. Meza-Junco J, Montano-Loza AJ, Baracos VE, et al. Sarcopenia as a prognostic index of nutritional status in concurrent cirrhosis and hepatocellular carcinoma. $J$ Clin Gastroenterol. 2013;47(10):861-70.

12. Voron T, Tselikas L, Pietrasz D, et al. Sarcopenia impacts on short- and long-term results of hepatectomy for hepatocellular carcinoma. Ann Surg. 2015;261(6):1173-83.

13. Hamaguchi $Y$, Kaido T, Okumura S, et al. Muscle steatosis is an independent predictor of postoperative complications in patients with hepatocellular carcinoma. World $J$ Surg. 2016;40(8):1959-68.

14. Fearon K, Strasser F, Anker SD, et al. Definition and classification of cancer cachexia: an international consensus. Lancet Oncol. 2011;12(5):489-95.

15. Cruz-Jentoft AJ, Bahat G, Bauer J, et al. Sarcopenia: revised European consensus on definition and diagnosis. Age Ageing. 2019;48(1):16-31.

16. Kobayashi A, Kaido T, Hamaguchi Y, et al. Impact of sarcopenic obesity on outcomes in patients undergoing hepatectomy for hepatocellular carcinoma. Ann Surg. 2019;269(5):924-31.

17. Smith AD, Zand KA, Florez E, et al. Liver surface nodularity score allows prediction of cirrhosis decompensation and death. Radiology. 2017;283(3):711-22.

18. Hectors SJ, Lewis S, Besa C, et al. MRI radiomics features predict immuno-oncological characteristics of hepatocellular carcinoma. Eur Radiol. 2020;30(7):3759-69.

Publisher's Note Springer Nature remains neutral with regard to jurisdictional claims in published maps and institutional affiliations. 